Www.jmscr.igmpublication.org

Impact Factor 5.84

Index Copernicus Value: 71.58

ISSN (e)-2347-176x ISSN (p) 2455-0450

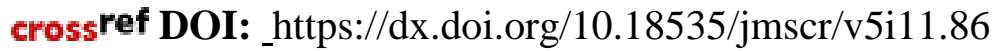

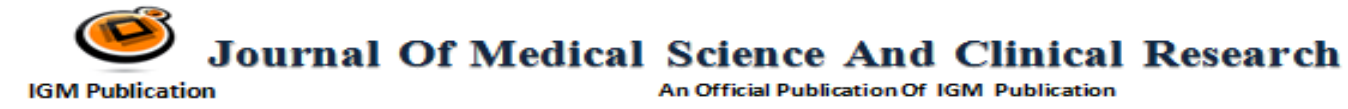

\title{
Anaesthesia for Neurosurgical Procedures in Paediatric Patients at Kamineni Institute of Medical Sciences
}

\author{
Authors \\ Dr Sujay ${ }^{1}$, Dr Santhosh Kumar Nangunuri \\ ${ }^{1}$ Assistant Professor, Anesthesia and Critical Care, Kamineni Institute of Medical Sciences Nalgonda, \\ Narketpalli, Telangana \\ Email:drsujaym@gmail.com \\ ${ }^{2}$ Senior Resident, Anesthesia and Critical Care, Kamineni Institute of Medical Sciences Nalgonda, \\ Narketpalli, Telangana \\ Email: santhoshkumar.n83@gmail.com \\ Corresponding Author

\section{Dr Sujay} \\ Address: JS Hospital, 1-14-158 Balamrai 68 Sappers Lines, Secunderabad 500003, Telangana State
}

PH: 09109190897, Email: mydoctorhyd@gmail.com

\begin{abstract}
Recent advances in neurosurgery, neuromonitoring and neurointensive care have dramatically improved the outcome in patients affected by surgical lesions of central nervous system (CNS). Although most of these techniques were applied first in the adult population, paediatric patients present a set of inherent challenges because of their developing and maturing neurological and physiological status, apart from the CNS disease process. To provide optimal neuroanaesthesia care, the anaesthesiologist must have the knowledge of basic neurophysiology of developing brain and effects of various drugs on cerebral haemodynamics apart from the specialised training on paediatric neuroanaesthesia. This article highlights on the perioperative management of paediatric neurosurgical patients.
\end{abstract}

\section{Introduction}

Recent advances in neurosurgery, neuromonitoring, and neurointensive care have dramatically improved the outcome in patients affected by surgical lesions of central nervous system (CNS). Although most of these techniques were applied first in the adult population, paediatric patients present a set of inherent challenges because of their developing and maturing neurological and physiological status, apart from the CNS disease process. This article highlights the anaesthetic management and postoperative care of paediatric neurosurgical patients.
Accurate data pertaining to normal neurophysiological variables in paediatric patients are limited and often derived from the adult and animal data. Cerebral blood flow (CBF) affects the cerebral blood volume and the intracranial volume, thereby affecting the intracranial pressure (ICP). It varies with age in paediatric population, being lower in premature infants $(12 \mathrm{ml} / 100$ $\mathrm{g} / \mathrm{min}$ ) and full-term neonates (23-40 $\mathrm{ml} / 100$ $\mathrm{g} / \mathrm{min}$ ) and higher in infants and older children than in adults $(50 \mathrm{ml} / 100 \mathrm{~g} / \mathrm{min}) .{ }^{[1],[2],[3],[4]}$ From 6 months to 3 years of age, $\mathrm{CBF}$ is $90 \mathrm{ml} / 100$ $\mathrm{g} / \mathrm{min}$; and from 3 to 12 years of age, the CBF is 
$100 \mathrm{ml} / 100 \mathrm{~g} / \mathrm{min}$. CBF is coupled tightly with the metabolic demand known as cerebral metabolic rate of oxygen $\left(\mathrm{CMRO}_{2}\right)$, and both increase proportionally after birth. In children, the $\mathrm{CMRO}_{2}$ is higher at $5.2 \mathrm{ml} / 100 \mathrm{~g} / \mathrm{min}$ than the adults $(3.5 \mathrm{ml} / 100 \mathrm{~g} / \mathrm{min})$ and hence, less tolerant to hypoxia. Neonates have a lower $\mathrm{CMRO}_{2}(3.5$ $\mathrm{ml} / 100 \mathrm{~g} / \mathrm{min}$ ) with a relative tolerance to hypoxaemia. ${ }^{[4]}$

The autoregulation range of blood pressure in normal newborn is narrow between 20 and 60 mmHg. ${ }^{[5]}$ The autoregulatory slope drops and rises significantly at the lower and upper limits of the curve, respectively. Sudden hypotension and hypertension at either end of the autoregulatory curve places the neonate at risk for cerebral ischaemia and intraventricular haemorrhage (IVH), respectively. The skull is a closed box with brain tissue, blood and cerebrospinal fluid (CSF) as its contents. An increase in volume of one of these components with increase in ICP will result a compensatory reduction of other components (Monro-Kellie doctrine). In infants, open fontanel and cranial sutures lead to a compliant intracranial space. The mass effect of a space-occupying lesion can be masked by an increase in skull size. Hence, infants presenting with intracranial hypertension $(\mathrm{ICH})$ may have a well-advanced pathology.A large percentage of cardiac output is directed to brain in infants and children as the head accounts for large percentage of the body surface area and blood volume. This aspect places the infant at risk for significant haemodynamic instability during neurosurgery.

\section{Preoperative Considerations}

\section{Preoperative evaluation and preparation}

The evaluation must include history and physical examination pertaining to the conditions requiring special anaesthetic considerations. Assessment of neurological status should include evidence of raised ICP, altered sensorium and cranial nerve palsies. Infants with $\mathrm{ICH}$ might present with irritability, lethargy, decreased consciousness, failure to feed, bulging fontanel and cranial enlargement. ${ }^{[6]}$ In children, it may present with early morning headache, vomiting without nausea, diplopia, and papilloedema, and in late stage, with Cushing's triad. Frequent vomiting episodes may lead to dehydration and electrolyte imbalances, and increase the risk of aspiration. Hence, serum electrolytes should be determined to identify abnormalities of sodium and potassium following vomiting. Other laboratory investigations should include haemoglobin or haematocrit level, and typing and cross-matching of blood if the loss is expected to be considerable. Additional studies should include electrocardiogram (ECG), coagulation profile, renal and hepatic function, as deemed necessary. Children with pituitary tumours should undergo complete endocrine evaluation.

\section{Premedication}

Sedative and narcotic premedication should be avoided in all the children suspected of increased ICP as these medications decrease respiratory drive which may result in hypercapnia and further increase in ICP. ${ }^{[7]}$ However, patients with normal ICP, such as those scheduled for repair of vascular lesions, may be sedated so as to allay preoperative anxiety and avoid hypertension, thus preventing rupture of vascular abnormality. Oral benzodiazepines (midazolam) may be beneficial for small children as they provide sedation without respiratory depression and should be administered under supervision.

\section{Induction}

The goal of anaesthetic induction is to avoid increase in ICP owing to associated hypoxia, hypercapnia and volatile anaesthetic-induced increases in $\mathrm{CBF} .{ }^{[8]} \mathrm{An}$ intravenous (IV) induction with thiopentone or propofol and neuromuscular block to facilitate endotracheal intubation is ideal in children with raised ICP. However, in children without IV access or with difficult IV access, inhalational induction by facemask with sevoflurane should be preferred as crying or struggling may lead to further increase in ICP. ${ }^{[7]}$ After the IV access is secured, a bolus of thiopentone $(1-2 \mathrm{mg} / \mathrm{kg})$ or propofol may be 
given to prevent the pressure responses of tracheal intubation. Furthermore, the inhalational technique may subsequently be changed to an IV induction. ${ }^{[6]}$ All volatile anaesthetics cause an increase in CBF, and thence the ICP. Therefore, ventilation should be controlled as early as possible and mild hyperventilation be instituted to prevent rise in ICP. Children at risk for aspiration should undergo rapid-sequence anaesthetic induction with thiopentone or propofol followed by rapid-acting muscle relaxants such as succinylcholine or rocuronium.

\section{Maintenance of anaesthesia}

Anaesthesia is maintained either with low endtidal volatile agents (minimum alveolar concentration $(\mathrm{MAC})<1$ ) or with total IV anaesthesia (TIVA), along with short-acting opioids (fentanyl or remifentanil), inhaled nitrous oxide and controlled ventilation. ${ }^{[9]}$ Sevoflurane has almost replaced halothane as an agent for induction; isoflurane, sevoflurane or desflurane is used for maintenance of anaesthesia during paediatric neurosurgical procedures. ${ }^{[9],[10]}$

Sevoflurane provides smooth induction followed by a rapid recovery. ${ }^{[10]}$ Neuromuscular blockade with non-depolarising muscle relaxants is given to prevent patient movement and minimise the amount of anaesthetic agent required. Children on chronic anti-convulsant therapy will require large doses of muscle relaxants and narcotics due to enzymatic induction of these drugs. Muscle relaxant should be withheld when assessment of motor function is carried out, e.g. during spinal cord surgery. Fentanyl is the most commonly used opioids, but its half-life increases with repeated dosing. It requires hepatic metabolism which is immature in premature infants. Hence, the sedative and respiratory depressive effects of fentanyl may be prolonged in these children. Remifentanil is unique in the sense it is cleared rapidly by the plasma esterases, but the associated rapid recovery may be accompanied by delirium and inadequate analgesia. Hence, it requires supplementation of other analgesics during postoperative period. Currently, it is not available in India.

\section{Positioning}

The neurosurgical procedures in children are carried out in different positions with certain challenges for each one of them. These surgeries are usually of prolonged duration during which the small child may disappear under the surgical drape and equipments. Hence, the anaesthesiologist must try to ensure unobstructed view of the part of child, proper padding of pressure points, protection of the eyes from surgical cleaning solutions, and the access to IV line, airway and breathing circuits before commencement of surgery.

Supratentorial surgeries and ventriculoperitoneal (VP) shunt surgery are carried out with the patient lying supine with head probably turned to one side. It is better to secure the endotracheal tube (ETT) in the non-dependant corner of the mouth to prevent oral secretions loosening the adhesive tapes used for fastening. ${ }^{[6]}$ The prone position is used during suboccipital craniotomy for posterior fossa lesions, spine surgery and repair of encephaloceles [Figure 1]. It must be ensured that the weight of the child is supported on bolsters under the chest and pelvis, and does not rest on the abdomen. Excessive pressure on the abdomen impedes ventilation, compresses vena cava and increases epidural venous pressure and bleeding. There may be congestion of face and tongue, and pressure sore on malar prominences owing to horseshoe head rest. ${ }^{[11]}$ Extreme flexion of the neck may cause endobronchial intubation because of short trachea ${ }^{[12]}$ and intraoral kinking of ETT; hence, armoured ETTs are preferred. Excessive flexion or extension of head may cause brainstem compression in patients with Arnold-Chiari malformation (ACM). ${ }^{[9]}$ The sitting position is still being practiced in few centres across the globe [Figure 2]. ${ }^{[13]}$ In children more than 4 years of age, it is used for the exploration of the posterior fossa with the advantage to reduce intraoperative bleeding and to facilitate surgical exposure. Complications in relation to this 
position include cardiovascular instability, venous air embolism (VAE) and postoperative tension pneumocephalus. Extreme flexion of neck required in this position may be complicated by mid cervical flexion myelopathy and macroglossia. Lateral position is rarely used in paediatric patients with ipsilateral posterior lesions or cerebellopontine angle tumours [Figure 3].

\section{Monitoring}

Intracranial surgery may be associated with haemodynamic changes owing to sudden blood loss, VAE and manipulation of cranial nerves. A part from the routine monitors, placement of an arterial cannula permits continuous monitoring of blood pressure, occasional estimation of arterial blood gas, electrolytes and haematocrit values. A central venous catheter provides large-bore access and aspiration of air during occurrence of VAE in sitting position. Precordial Doppler and transoesophageal echocardiography are sensitive monitors used to detect of VAE, but not available everywhere. Hence, the end-tidal $\mathrm{CO}_{2}$ is commonly used in India for this purpose. ${ }^{[13]}$

Neurophysiological monitoring may be used with the goal to reduce morbidity by early detection of neurological insult when it is reversible, thereby improving the overall outcome. The monitoring modalities include electroencephalography (EEG), sensory evoked potentials (SSEP), motor evoked potentials (MEP) and brainstem auditory evoked potentials (BAEP).

\section{Fluid management}

The intraoperative goal is to maintain normovolaemia, and thus the haemodynamic stability. Normal saline is the most commonly administered crystalloid during paediatric neurosurgical procedures as it is mildly hyperosmolar and hence prevents cerebral oedema. ${ }^{[6]}$ However, infusion of large quantities $(>60 \mathrm{ml} / \mathrm{kg}$ ) of normal saline may cause hyperchloraemic metabolic acidosis and hypernatraemia. ${ }^{[14]}$ Ringer's lactate is slightly hypo-osmolar and infusion of large quantities may increase cerebral oedema. Glucose-containing fluids should not be used during these procedures as hyperglycaemia worsens reperfusion injury. However, in neonates and premature infants, the danger of hypoglycaemia should be borne in mind. Blood glucose should be closely monitored in these patients, along with continuous infusion of glucose at 5-6 mg/kg/min. ${ }^{[9]}$ Children do not need exogenous glucose administration and are able to maintain normal levels along with the associated surgical stress. Blood transfusion should be guided by the degree of blood loss and initial haematocrit values.

\section{Temperature management}

Although the experimental animal models favourably suggested the use of mild hypothermia in neurosurgical patients, it has not been extrapolated to humans. The associated complications prevent use of hypothermia in routine paediatric neurosurgical practice despite obtaining encouraging results following brain injury. ${ }^{[15]}$ The intraoperative goal is to maintain normothermia and avoid both hypothermia and hyperthermia with application of different methods.

\section{Recovery and postoperative care}

The goal is "rapid awakening" in order to help early neurological assessment, haemodynamic stability, and minimal coughing and straining in the ETT. Sevoflurane provides better recovery profile, as compared to isoflurane in children. ${ }^{[10]}$ Trachea is extubated once the child responds to commands or infants open their eyes. However, there are circumstances where the children continue to remain intubated during the postoperative period. Surgeries that interfere with cranial nerve nuclei and brainstem function with depressed respiratory drive require postoperative mechanical ventilation till these functions are improved. ${ }^{[16]}$ Generally, the intracranial procedures require close observation in the intensive care unit (ICU). Children undergoing extracranial or minor procedures may be nursed in an inpatient unit (ward). 


\section{Brain tumours}

These are most common solid tumours of childhood, two-thirds of which arise from the infratentorial compartment. Management of these posterior fossa tumours poses unique challenges to the neuroanaesthesiologist in the form of $\mathrm{ICH}$ due to obstructive hydrocephalus, the pressure responses to laryngoscopy and Mayfield head-pin fixation in children with deranged intracranial compliance, different positions (e.g. sitting position) and their related problems, and possible respiratory and cardiovascular changes during brainstem manipulations. Damage to respiratory centres and lower cranial nerves may cause apnoea and airway obstruction in the postoperative period. ${ }^{[13]}$ The head-pin fixation in children may cause skull fracture, dural tears, and even haematoma at pin sites. ${ }^{[23]}$

In children, craniopharyngiomas are the most common tumours in the perisellar region. These tumours are associated with hypothalamopituitary dysfunction and require perioperative steroid replacement therapy. The children may present with endocrine abnormalities; preoperative thyroid and adrenal function should be obtained. Diabetes insipidus (DI) may occur during preoperative (8-35\%), and rarely in intraoperative period; but is quite common (70$90 \%)$ in the postoperative period. ${ }^{[24]}$ DI causes large volume of urine which needs to be replaced on hourly basis. If the resultant hypovolaemia is not corrected with fluids, DI must be treated pharmacologically with synthetic vasopressin (DDAVP).

\section{Meningomyelocele and encephalocele}

Embryogenic neural tube fusion occurs during the first 4 weeks of gestation. Failure of fusion causes herniation of meninges with (meningomyelocele (MMC)) or without (meningocele) neural elements in any level of the spinal cord. When this neural tube defect occurs in cranium, the meninges protrude through a bony defect on skull with (encephalocele) or without (cranial meningocele) brain tissue. All these defects require correction within first few days of life.
MMC is usually associated with $A C M$ (most commonly Type II) and hydrocephalus. ${ }^{[12]}$ The characteristics of ACM include downward displacement of cerebellar vermis into upper cervical spinal canal, and elongation of brainstem and fourth ventricle. ${ }^{[7]}$ These children may present with features of brainstem compression such as apnoea, vocal cord palsy causing stridor, ${ }^{[7],[25]}$ autonomic instability and abnormal respiration. These children require surgical decompression of posterior fossa to accommodate the hind brain malformation.

Inhalational induction with sevoflurane is the commonly practiced technique as most of the children come to the operating room without an IV access. Tracheal intubation is carried out with the child in supine position and the swelling is supported on a doughnut, or in case of large $\mathrm{MMC}$, in lateral decubitus position. Excision and repair of MMC in prone position is combined with insertion of a VP shunt for hydrocephalus in supine. ${ }^{[12]}$ The spinal cord below the defect of MMC is often tethered, which over a period of time results in distal neurological defects, scoliosis or kyphoscoliosis. Untethering of the cord needs Electromyography (EMG) monitoring to identify functional nerve roots. During the procedure, there may be sudden blood loss from an abnormal vessel or occurrence of VAE. ${ }^{[26]}$ These children have an increased risk of latex allergy, although it is rarely encountered in Indian context. ${ }^{[12]}$

Occiput is the most common site for the occurrence of encephaloceles [Figure 1], ${ }^{[27]}$ although fronto-ethmoidalencephalocele is quite common in South-East Asia. ${ }^{[28]}$ There may be problems in mask ventilation and potential rise in ICP due to compression of the sac during various manipulations in children with anterior encephalocele. ${ }^{[29]}$ Children with posterior encephalocele are intubated commonly in lateral decubitus position, or with the swelling supported by a doughnut, and even by placing the child's head beyond the edge of the table, supported by an assistant. Our experience suggests that placing the child in right lateral position helps in getting more 
space for laryngoscopy and intubation for a righthanded anaesthesiologist and vice versa. ${ }^{[30]}$ Intraoperative haemodynamic disturbances are commonly encountered during encephalocele repair. Apart from having a strong vagal tone, children with Chiari malformation may experience bradycardia during laryngoscopy and intubation due to compression of the brainstem. However, an event of cardiac arrest during these manipulations should not deter the neuroanaesthesiologist to continue with the procedure. ${ }^{[31]}$

\section{Epilepsy surgery}

Surgical treatment offered to children with medically intractable epilepsy includes focal resection (of the area generating seizures), corpus callosotomy (two hemispheres surgically separated to reduce severity of seizures), hemispherectomy (disconnecting one side of the brain from rest) and vagal nerve stimulation (timed stimulation of left vagus nerve to blunt paroxysmal seizures).

The anaesthetic concerns include perioperative seizures and the effects of anticonvulsants. Chronic use of anticonvulsants induces rapid metabolism and clears anaesthetic agents such as neuromuscular blockers and opioids, thereby increasing the anaesthetic requirements. If intraoperative electrocorticographic (ECoG) monitoring is planned, sedatives and anticonvulsants should be withheld for $48 \mathrm{~h}$ preoperatively. ${ }^{[16]}$ Inhalational agents depress the cortical responses, hence should be avoided during ECoG recordings. An opioids-based technique is ideal during such a scenario. Awake craniotomy offers the advantage of intraoperative ECoG monitoring, but is difficult to get the cooperation of children below 9 years of age. ${ }^{[32]}$

\section{Craniovertebral junction anomalies}

Craniovertebral junction (CVJ) anomalies are developmental disorders that affect the skeleton and enclosed neuraxis at the junction of cranium and cervical spine. The clinical syndromes associated with these anomalies are attributable to the following: (i) pressure on the neuraxis by the bony abnormalities, (ii) intrinsic malformations of the nervous system and (iii) disturbance of the CSF circulation and blood supply. ${ }^{[33]}$ The bony abnormalities usually encountered in CVJ anomalies are basilar invagination, occipitalisation and congenital atlanto-axial dislocation. The clinical manifestations may occur because of direct compression of the neural tissue by these osseous anomalies as the spine is grossly unstable. The patients may present with respiratory dysfunction (restrictive lung disease) owing to compression of the brainstem by odontoid process [Figure 6] of second cervical vertebra affecting the respiratory centre and weakening of the muscles of respiration including diaphragm. ${ }^{[34]}$ The surgical management includes transoralodontoidectomy and occipito-cervical fixation, with both the procedures carried out in one setting. The anaesthetic considerations include securing the airway with minimal manipulation of neck, preferably by awake fibreoptic intubation, blood loss during odontoidectomy, haemodynamic changes during posterior fixation owing to prone position and handling near cervico-medullary junction. Postoperatively, the tracheal intubation is continued for at least $12-24 \mathrm{~h}$ to prevent complications of potential reintubation due to pharyngeal oedema ${ }^{[35]}$ with a fixed cervical spine.

\section{Vascular malformations}

Arteriovenous malformations (AVMs) present with haemorrhage, seizure and hydrocephalus in infants and children, and with congestive heart failure (CHF) in newborns. ${ }^{[36]}$ AVMs are the most common cause of subarachnoid haemorrhage in children. Large AVMs, e.g. vein of Galen malformation, may present with high-output CHF. Usually intravascular embolisation is carried out in a neuroradiological suite as surgical excision is associated with considerable blood loss requiring adequate haemodynamic monitoring. The anaesthesiologist should be prepared to treat sudden hypertension and hyperaemic cerebral oedema following embolisation/excision of AVM with labatolol and sodium nitroprusside. 
Moyamoya disease is a vaso-occlusive disorder of the distal internal carotid artery, present in children with transient ischaemic attacks or recurrent strokes. Surgery for this condition, encephalo-duro-arterial-synangiosis (EDAS), may be often complicated by cerebral ischaemia. Hence, the anaesthetic goal is to optimise cerebral perfusion pressure (CPP) by normovolaemia with preoperative hydration, normotension, normothermia and normocapnia, ${ }^{[37]}$ as both hypo and hypercapnia cause steal phenomena from the ischaemic region, thereby causing further cerebral ischaemia.

\section{Neuroradiology}

Most of the neuroradiological studies (computed tomography (CT) scan and magnetic resonance imaging (MRI)) can be carried out with light sedation. However, general anaesthesia (GA) is required in children who are uncooperative with co-existing medical conditions and during the procedure of intravascular embolisation which may be painful for a child. Endotracheal intubation is not always required to secure the airway for the children undergoing these procedures under GA. Use of laryngeal mask airway has been suggested, ${ }^{[4],[38],[39]}$ although there is insufficient evidence to recommend its routine use.

\section{Head trauma}

Head injury may cause intracranial haematoma, diffuse axonal injury and oedema with deranged autoregulation and intracranial compliance. Sometimes, the children with head trauma may present with depression of ST segment in the ECG. ${ }^{[40]}$ These ST-T changes are possibly due to sympathetic hyperactivity associated with raised ICP and should not deter the anaesthesiologist to continue with the anaesthetic plan. These changes revert back to normal once the decompressive procedure is carried out. The anaesthetic goal is to prevent secondary injuries such as hypoxia, hypotension, hyperthermia, hyperglycaemia, hypoglycaemia, etc., Cross-matched blood must be available during emergency evacuation of an extradural haematoma. In a child, a small intracranial bleed may result in significant loss of circulating volume. Subsequent hypovolaemia and hypotension may be further aggravated by preoperative infusion of mannitol and diuretic therapy. Hence, precaution should be taken to prevent hypotension even it occurs for a brief time period.

\section{Conclusion}

Anaesthesia for children undergoing neurosurgery requires knowledge on cerebral pathophysiology and the interaction of anaesthetic agents on brain. The inherent problems of paediatric population pose a unique challenge to the anaesthesiologist and its management depends on specialised training on paediatric neuroanaesthesia and cooperation from the neurosurgeons.

\section{Reference}

1. Singh D, Rath GP, Dash HH, Bithal PK. Sevoflurane provides better recovery as compared with isoflurane in children undergoing spinal surgery. J Neurosurg Anesthesiol 2009;21:202-6. [PUBMED]

2. Singh D, Rath GP, Dash HH, Bithal PK. Anesthetic concerns and perioperative complications in repair of myelomeningocele: A retrospective review of 135 cases. J Neurosurg Anesthesiol 2010;22: 11-5. [PUBMED]

3. Rath GP, Bithal PK, Chaturvedi A, Dash HH. Complications related to positioning in posterior fossa craniectomy. $\mathrm{J}$ Clin Neurosci 2007;14:520-5. [PUBMED]

4. Scheingraber S, Rehm M, Sehmisch C, Finsterer U. Rapid saline infusion produces hyperchloremic acidosis in patients undergoing gynecologic surgery [see comments]. Anesthesiology 1999;90:1265-70. [PUBMED]

5. Grinkeviciute D, Kevalas R. Induced mild hypothermia in children after brain injury. Rev Neurosci 2009;20:261-6. [PUBMED]

6. Newfield P, Feld LH, Hamid RK. Induced mild hypothermia in children after brain 
injury. In: Newfield P, Cottrell JE, editors.

Philadelphia: Lippincott Williums and Wilkins; 2007. p. 256-77.

7. Singh GP, Prabhakar H, Bithal PK, Dash HH. A retrospective analysis of perioperative complications during intracranial neuroendoscopic procedures: Our institutional experience. Neurol India 2011;59:874-8.

8. Prabhakar H, Rath GP, Bithal PK, Chouhan RS. Intracranial pressure and haemodynamic changes during the tunnelling phase of ventriculoperitoneal shunt insertion. Eur $\mathrm{J}$ Anaesthesiol 2005;22:947-50. [PUBMED]

9. Rath GP, Prabhakar H, Bithal PK, Dash HH, Narang KS, Kalaivani M. Effects of butorphanol and fentanyl on cerebral pressures and cardiovascular hemodynamics during tunneling phase for ventriculoperitoneal shunt insertion. Middle East J Anesthesiol 2008;19:104153. [PUBMED]

10. Alfery DD, Shapiro HM, Gagnon RL. Cardiac arrest following rapid drainage of cerebrospinal fluid in a patient with hydrocephalus. Anesthesiology 1980;52:443-4. [PUBMED]

11. Faberowski LW, Black S, Mickle JP. Incidence of venous air embolism during craniectomy for craniosynostosis repair. Anesthesiolgy 2000;92:20-3. [PUBMED]

12. Goyal K, Chaturvedi A, Prabhakar H. Factors affecting the outcome of patients undergoing corrective surgery for craniosynostosis: A retrospective analysis of 95 cases. Neurol India 2011;59:823-8.

13. Soriano SG, Eldredge EA, Rockoff MA. Pediatric neuroanesthesia. Anesthesiology Clin N Am 2002;20:389-404. [PUBMED]

14. Ghiarardello S, Hopper N, Albanese A, Maghnie M. Diabetes insipidus in craniopharyngioma: Postoperative management of water and electrolyte disorders. J
Pediatr Endocrinol Metab 2006;19Suppl 1:413-21.

15. Rath GP, Bithal PK, Chaturvedi A. Atypical presentations in Chiari II malformation. Pediatr Neurosurg 2006;42: 379-82. [PUBMED]

16. Kennedy C, Sokoloff L. An adaptation of the nitrous oxide method to the study of the cerebral circulation in children; normal values for cerebral blood flow and cerebral metabolic rate in childhood. J Clin Invest 1957;36:1130-7. [PUBMED]

17. Chiron C, Raynaud C, Maziére B, Zilbovicius M, Laflamme L, Masure MC, et al. Changes in regional cerebral blood flow during brain maturation in children and adolescents. J Nucl Med 1992;33:696703.

18. Krass IS. Physiology and metabolism of brain and spinal cord. In: Newfield P, Cottrell JE, editors. Handbook of Neuroanaesthesia. Philadelphia: Lippincott Williams and Wilkins; 2007. p. 3-22.

19. Pryds O. Control of cerebral circulation in the high-risk neonate. Ann Neurol 1991;30:321-9. [PUBMED]

20. Krane EJ, Phillip BM, Yeh KK, Domino KB. Anaesthesia for paediatric neurosurgery. In: Smith RM, Mototyama EK, Davis PJ, editors. Smith's Anaesthesia for Infants and Children, 7 th Edn. Philadelphia: Mosby; 2006. p. 651-84.

21. Pollard RJ, Mickle JP. Pediatric neuroanesthesia. In: Cucchiara RF, Black $\mathrm{S}$, michenfelder JD, editors. Clinical Neuroanesthesia. Newyork: Churchil Livingstone; 1998. p. 497-537. 